\title{
Design Alternatives for a MediaWiki to Support Collaborative Writing
}

\author{
Sumonta Kasemvilas and Lorne Olfman \\ School of Information Systems and Technology, \\ Claremont Graduate University, Claremont, CA, USA
}

\section{Sumonta.Kasemvilas@cgu.edu; Lorne.Olfman@cgu.edu}

\begin{abstract}
Web 2.0 technologies are having a considerable influence on the learning industry. Constructivist learning processes, such as collaborative writing, have emerged as a result of the development of Web 2.0 technologies. We define the term "mandatory collaborative writing" to describe a writing task where the group has a firm deadline. This article focuses on how a wiki can fully support mandatory group writing. The motivation of this design science research study emerges from a graduate Knowledge Management class assignment to write a wiki book. The main finding shows that the wiki instance used for the project, MediaWiki, could better facilitate the collaborative writing process with a set of extensions that support discussion and project management. We outline designs for these processes: 1) a discussion process that changes the way users discuss content on a wiki page and increases group awareness; and 2) a project management process that increases awareness of the status of each component of the writing project and provides an overall summary of the project. A demonstration of the principles to a focus group provided a basic proof of the validity of this design. The significant contributions of this study are these processes, which can facilitate group writing and constructivist learning processes. Contributions of the study to several streams of research are discussed.
\end{abstract}

Keywords: Awareness, collaborative writing, constructivist learning, design science research, discussion, MediaWiki, project management, transdisciplinary, Web 2.0

\section{Introduction}

Wikis can be used to facilitate collaborative writing. Through the use of a wiki for a group writing project we discovered that some processes can be added to wikis to support collaborative group writing in higher education. Collaborative writing can be used for varied purposes, which we term mandatory, optional, and hybrid. Based on the success of Wikipedia, it is generally assumed that a wiki is an effective collaborative writing tool. However, Wikipedia supports an optional collaborative writing purpose. Members of the Wikipedia community are voluntarily in-

Material published as part of this publication, either on-line or in print, is copyrighted by the Informing Science Institute. Permission to make digital or paper copy of part or all of these works for personal or classroom use is granted without fee provided that the copies are not made or distributed for profit or commercial advantage AND that copies 1) bear this notice in full and 2) give the full citation on the first page. It is permissible to abstract these works so long as credit is given. To copy in all other cases or to republish or to post on a server or to redistribute to lists requires specific permission and payment of a fee. Contact Publisher@InformingScience.org to request redistribution permission. volved in a project that does not have deadlines or specific goals that the project needs to achieve. In contrast, a mandatory purpose in this context is one in which a group must finish its project within a specific timeline. Examples of this type of collaborative writing are class projects and responses to requests for proposals. Both these purposes require a different project management style. In the mandatory case, project 
management must be formally applied or the deadline will not be met. In the optional case, there is no necessity for formal project management. A hybrid purpose, which has characteristics of the other two, is one where a project can be considered completed but with no real deadline. Examples of this hybrid purpose are a white paper being authored by a Community of Practice (CoP) and a set of requirements for an open source software project. We address mandatory collaborative writing in higher education in the paper.

Cunningham (2006) created the concept of a wiki. He specified a number of characteristics that he envisioned as necessary for enabling collaborative knowledge sharing. In this paper, we report on the problems encountered in using MediaWiki (one of the most popular wiki engines) in a classroom-based collaborative writing project. First, we review the literature on constructivist learning. Next, we compare the needs of the mandatory and optional purposes with the goals that Cunningham specified. Then, we provide a description of the course and its processes. We show that a wiki has the potential to support mandatory purposes, but requires additional facets to do so effectively. Next, we define the objectives for a solution and propose two processes for MediaWiki to provide aid for discussion and project management functionality and to better support mandatory collaborative writing. The significant contributions of this study are these processes, which can facilitate group writing and constructivist learning processes. We describe the design of these two processes and explain how they support group writing and constructivist learning processes. Then, we conduct a preliminary demonstration and evaluation. The paper concludes with limitations and proposed plug-ins development and testing.

\section{Research Question}

Current wiki technology has several challenges in the way it supports collaborative writing, such as how group members delegate responsibilities and how they coordinate with each other. Therefore, we posed the following question: Can we design processes to facilitate collaborative group writing in a classroom setting? To answer this question, we used our experience from a class that used MediaWiki as a mandatory collaborative writing tool using a design science methodology.

\section{Design Science Research}

According to Hevner, March, Park, and Ram (2004, p. 79), "design science addresses research through the building and evaluation of artifacts designed to meet the identified business need." This study follows six steps of design science research methodology (DSRM): 1) problem identification and motivation; 2) defining the objectives for a solution; 3) design and development; 4) demonstration; 5) evaluation; 6) communication (Peffers, Tuunanen, Rothenberger, \& Chatterjee, 2007). The purpose of the study is to design useful processes for groups who are writing wiki pages and need tools to support discussion and collaboration. Researchers have designed a supporting library of tools and defined taxonomies to address some of these requirements, but these mechanisms are still in an evolving stage. A number of studies have attempted to solve the problems inherent in wiki technology, especially in Wikipedia (B. Suh, Chi, Kittur, \& Pendleton, 2008). This paper will focus on wiki collaborative writing among graduate students because collaborative writing is important in many graduate programs.

\section{Problem Identification and Motivation}

The second author of this paper designed a graduate Knowledge Management course in which the goal was to write a textbook as the lone class assignment. The first author of this paper was one of the students in the class. The writing platform for the group writing project was a MediaWiki instance. In this section, we discuss the concept of constructivist learning that views learning as an active, social process. Given this approach, we suggest that group writing is an appropriate task for facilitating constructivist learning. Then we justify the choice of using a wiki, and spe- 
cifically, MediaWiki. Finally, we describe the details of the class project, and the outcomes of the project in terms of the quality of the book, some feedback from students as to their learning experiences, the effectiveness of MediaWiki for the task, and the technical problems discovered in writing process.

\section{Constructivist Learning}

Constructivism is a paradigm of learning. The most important concept in constructivism is a strong focus on student-centered learning. Constructivist learning theory considers the learner as a unique and complex individual who is actively involved in a collaborative learning process (Bruner, 1996; Dewey, 1916; Vygotsky, 1978). This theory views learning as an active, social process (Bruner, 1996; Dewey, 1916; Vygotsky, 1978). Thus, emerging knowledge is socially produced by the learner. In a constructivist learning environment, a student will actively participate with other students to learn together (Harasim, 1990). The learner is the focal point, as opposed to the lesson plan or subject matter that the instructor will teach. Learning occurs in realworld settings, and not in prearranged settings constructed by the instructor. According to Dewey (1916), rather than passively receiving knowledge from the instructor, the active learner needs to do activities to construct knowledge. The instructor needs to provide reflective activities, such as case-based learning or hands-on experience, to engage the minds of the learners in the learning process (Dewey, 1910). However, it is also important to the instructor to assure that the new information provided to learners is in an appropriate form and matches their level of understanding.

This theory also posits that learning takes place through social interaction and communication with others (Vygotsky, 1978). Success or failure depends on whether the learner feels more involved or hesitant to become closely involved with the group (Wegerif, 1998). The learner will construct knowledge by expressing ideas into words and sharing them with other learners and through negotiations among them. As learners may have different backgrounds and their own individual framework, when they collaborate with others, they can perceive a problem or a set of tasks from various different perspectives. They might agree, share, explore, negotiate, and create meanings through shared understanding amongst their group. Responses and reactions to these ideas from other members and group collaboration tasks assist the student in constructing knowledge (Alavi, 1994; Bouton \& Garth, 1983). According to Hiltz, Coppola, Rotter, and Turoff (1999, p. 106), "learning is not only active but also interactive."

Thus, in the classroom setting, the role of the instructor is to lead and conduct discourse with real, challenging projects, allowing students to engage new information with their own knowledge construction schema through communication and collaboration with their classmates. The whole time, the instructor guides and helps students to refine their understanding until it matches with the meaning of the material. The students take responsibility for their own learning by developing their own metacognitive abilities to organize themselves in the learning process.

\section{Wiki Technology}

Cunningham created the first wiki in 1994 because he found that programmers had difficulties exchanging their ideas. Cunningham wanted to create a simple online database and let the community help each other to create contents. According to Cunningham (2006), "[a] wiki is a work made by a community" (p. 6). The original design of the wiki was geared so that everyone can edit whatever whenever they want if they feel like the content does not fit for them (Richardson, 2006). Some wiki implementers have added additional concepts to the original principles that Cunningham created, including sharing, interaction, collaboration, and social network principles ("Wiki Design Principles," n.d.). Based on a recent white paper from the University of Delaware (2008) we assume that wiki technology can be used to support learning in higher education classes. The report introduces the idea of a Hybrid Wiki that includes features beyond those im- 
plemented by Cunningham. In the Hybrid Wiki, no users are anonymous, content is not made public, access is limited through various sets of permissions, copyright protections are maintained, contents include multimedia, contents are moderated by experts, and it there is a time limit for creating content.

Learning is not an immediate process. Learners need to revisit their understandings, contemplate on what they have learned, investigate their knowledge, and apply it. Learning is the process of going over one's own individual experiences and thoughts. Learners can take a long time to reach a moment of insight. Because of this, wiki technology is an appropriate technology to use in constructivist learning. Several features of wiki technology can support constructivist learning and collaborative writing. When learners gain new knowledge, they can come back to modify what they have previously written in a wiki. They can read what other users write, generate meaning through shared comprehension, refine and reshape their perceptions, and share or negotiate their understandings with other users.

Wikis can be used to assist teaching and learning (Parker \& Chao, 2007; Raman, Ryan, \& Olfman, 2005). However, because of the specific needs of instruction, assignments, and evaluation, classroom wikis need to be designed and used in a different way from Cunningham's original wiki design, which allowed anyone to edit a wiki however and whenever they pleased, and to do so anonymously. When we apply the wiki paradigm in a classroom environment, instructors have to formulate an evaluation model for fairness of individual and group assessments. Students need to finish group assignments based on instructors' requirements and within a limited timeframe. Students need to know their specific responsibilities, how they interact and collaborate with others, and their own progress as well as that of others. Instructors may need to act as "Editor-inChief" to manage scope, schedule, evaluation, and quality of contents (De PeDro et al., 2006). From this point of view, wikis that support mandatory collaborative writing cannot be open or arbitrary. Both students and instructors need to have specific roles and have to finish their tasks in a certain time period.

\section{Why MediaWiki}

MediaWiki was chosen as the platform for the class writing project because it is one of the world's most popular wikis- Wikipedia runs on MediaWiki - and it is easy to install, configure, and use. According to Barrett (2009), there are more than 2,000 wiki sites that use MediaWiki. These include Wikimedia projects such as Wiktionary, Wikisource, and Wikibooks. The option of moving the class project into the Wikibook space was also a motivation for choosing MediaWiki. Further, MediaWiki has a wide assortment of features, is very adaptable, and is scalable. It is a free and open source software platform, is very stable, has a mature development status, has an active community of developers, and can support 140 languages.

\section{Description of the KM Class Project}

Students taking a graduate class in knowledge management (KM) were required to write and edit an introductory textbook on the subject. This was the only assignment for the course. The class was composed of six males and three females. All students were familiar with using the Internet and some of them had used wikis before. The MediaWiki instance ran on a third-party server with restricted access. Students were motivated because the project was carried out in a class setting and their grades depended on their performance in writing and editing. Each student chose what major topics he or she wished to be responsible for writing in the KM book. The plan was for each student to finish one chapter of the book within the time limit of the semester. The "Main Page" of the book contained a detailed outline of the book chapters (see Figure 1). 


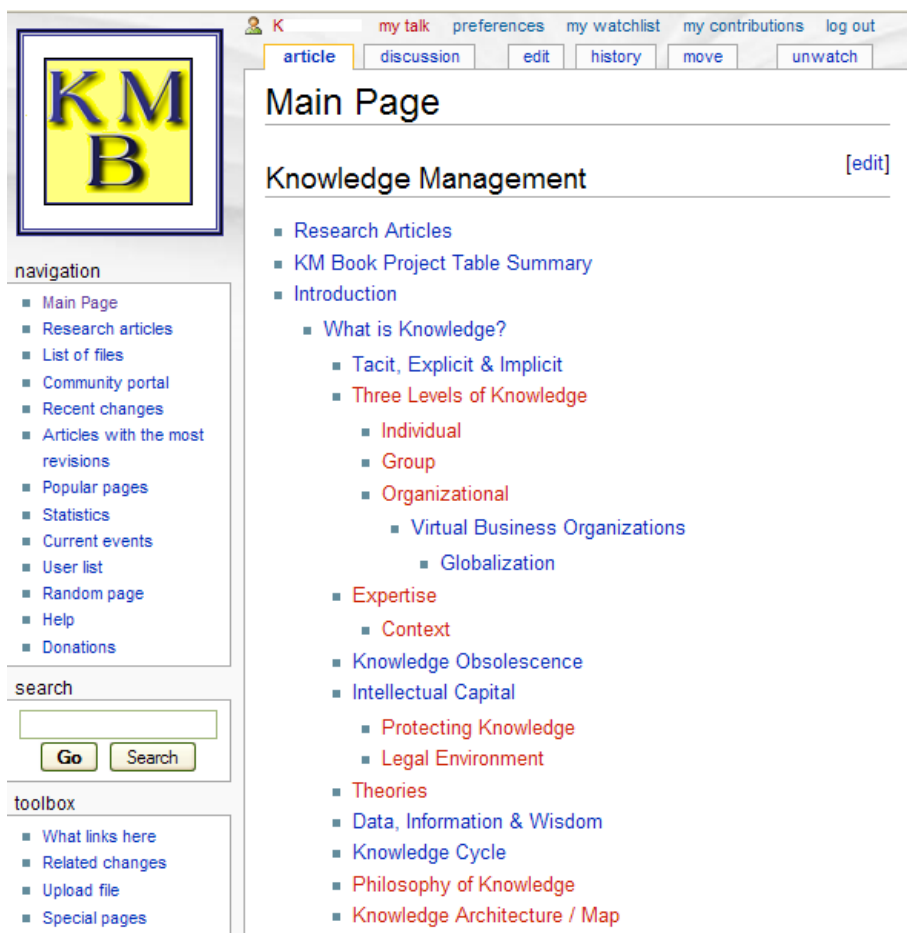

Figure 1: KM book main page

Editing roles were distributed across the group of nine students, with each student acting as Senior Editor each week and students in pairs acting as Associate Editors assigned to review 1/4 of the emerging content. The instructor acted as Editor-in-Chief. There were four roles (see Figure 2):

1. Writer: As a writer, a student was the first author on some pages of the book, and a secondary author on other pages. The pages each student contributed to as first author were ones that he or she expressed interest in writing about. Students could contribute to other pages as desired, with the possibility that his or her inputs would be first placed on the discussion page (in MediaWiki, there is a separate space for discussions of the content of pages) when it was not clear whether they fit closely with the writing that had already been contributed by the first author of that page. At times, the writer role and editor roles overlapped.

2. Editor-in-Chief: The Editor-in-Chief was the course instructor. He oversaw the writing process by working with the senior editors to assess the state of the book at the end of each week. He could make contributions as desired, but rarely did. The Editor-in-Chief made final decisions on debated issues, including ensuring that the book had a common look and feel. He discussed major decision items with the class members each week.

3. Senior Editor: The Senior Editor worked with the Editor-in-Chief and Associate Editors (pairs) to ensure the growth and development of the book. The Senior Editor ensured that the Associate Editors thoroughly edited their assigned portions of the book and provided an overall report of the book to the Editor-in-Chief. The Senior Editor asked the Editorin-Chief for advice on debated issues. The Senior Editor could make changes to pages or make suggestions to revise and/or change pages. Suggestions were documented on the discussion pages. 
4. Associate Editor: Associate Editors worked in pairs to edit (carefully read, revise, and suggest additions and changes to) specific wiki pages. Working in pairs meant that students must find concurrent time to go over the sections of the book that they were required to edit. Changes could be made directly on the wiki pages; suggestions had to be documented on the discussion pages. These may lead to debates about revisions.

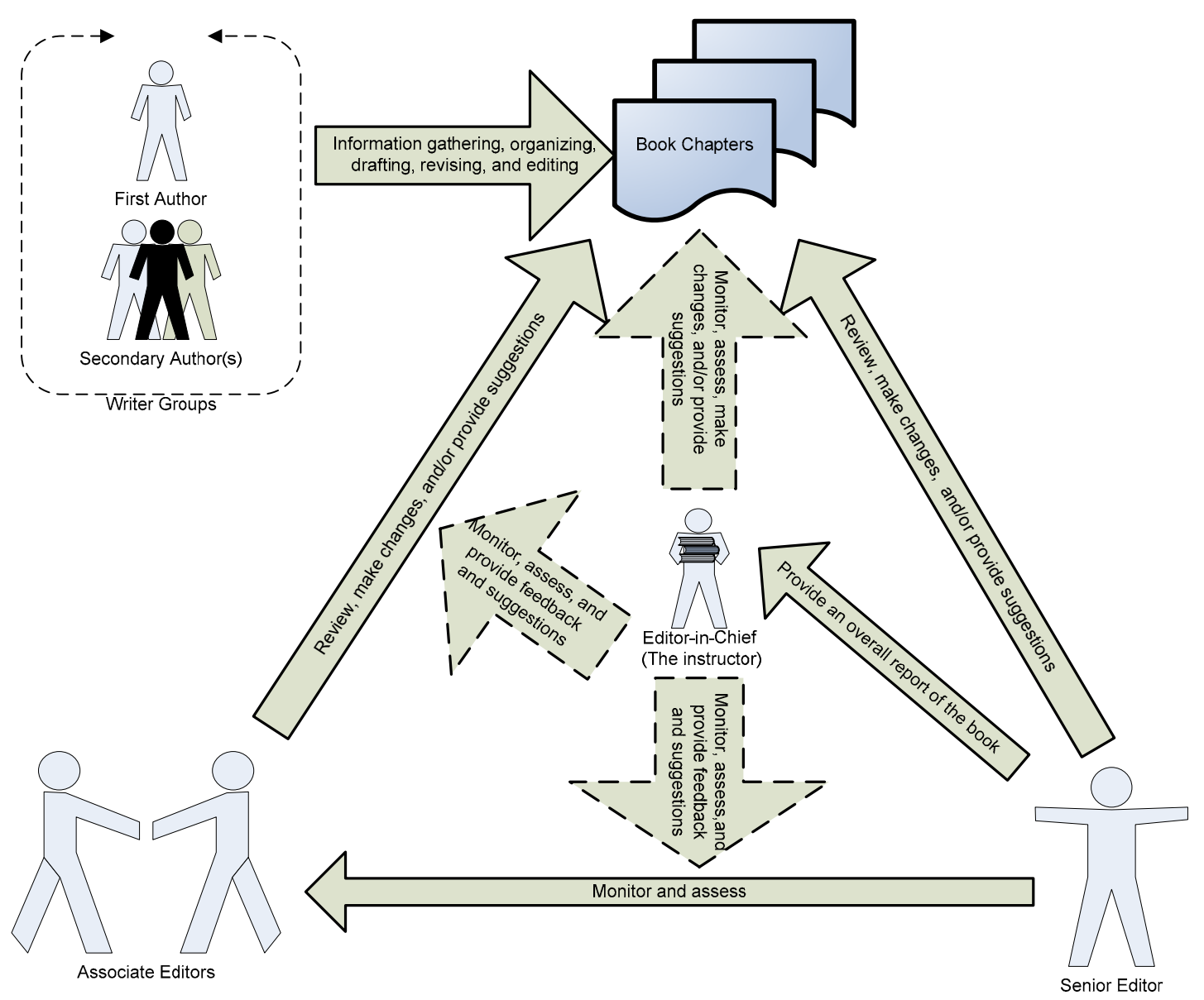

Figure 2: Model of collaborative writing in this study

While students were developing the content in the KM book, the Web site was open only for their use. The first few weeks of the class, the instructor provided a grade each week and feedback to students on what they should improve. The hope was that the students would produce good quality content, which could be made public after the class ended.

The instructor evaluated each student's contributed content and its quality. The instructor assessed students' performance by considering two rating categories for each page. First, information: the instructor evaluated what the page contributed to the subject it discussed, i.e., how the information in the page added to the detail of the book. The instructor used a 5-point scoring system to rate the quality of the information. A score of 3 meant that the information was basically not useful and could not be improved (it would have to be rewritten); a score of 7 meant that the information was minimally useful and could not be easily improved. A score 12 was allocated if the page needed further work but had the potential to contribute to the overall book content. A score of 15 meant that the page already had effective content but could be further improved; a 
score of 17 meant that the page was already publishable. Second, quality: the instructor evaluated the quality of each page and how ready it was for publishing. Quality was scored on a scale from 1 to 3 . A score of 1 meant that the page had many typos, formatting issues, etc. A score of 2 meant that page had a few typos and/or formatting issues. A score of 3 meant that the page had minimal typos and no formatting issues. The total score on each page was derived by adding the information score and the quality score. The overall range was 4 to 20. Additionally, the total score was factored by the number of words so that the greater the length, the higher the overall information score. The instructor also considered the priority of the primary author. A student who was the primary author of information would get $100 \%$ credit if there was no secondary author in that topic. If a student was a primary author and there were other authors in that topic, the primary author got $80 \%$ of the full score, while other students who contributed to existing content shared the remaining $20 \%$.

\section{Project Outcome}

As noted above, each of the nine students was responsible for writing about one of nine major topics. The amount of writing is summarized in Table 1 . There were 90 pages created in the wiki, with a total of 40,633 words (including references). More than half the pages received a total score of 19 or 20, showing that the students did a highly credible job of writing (information, words, quality and primary authorship being the factors that were used for this calculation). While all the students contributed to the KM book project, two of the students did almost $50 \%$ of the work.

\begin{tabular}{|l|r|}
\hline \multicolumn{2}{|c|}{ Table 1: The results of KM book project } \\
\hline \multicolumn{1}{|c|}{ Project Outcome } & \multicolumn{1}{|c|}{ Value } \\
\hline $\mathrm{n}$ (number of students) & 90 \\
\hline Number of pages in the wiki & 40,633 \\
\hline Total words created & 15.88 \\
\hline Average information score & 2.21 \\
\hline Average quality score & 452 \\
\hline \multicolumn{2}{|c|}{ Number of words per page } \\
\hline Average & 518 \\
\hline Standard deviation (SD) & 17 \\
\hline Minimum & 3,650 \\
\hline Maximum & 280 \\
\hline Median & \\
\hline
\end{tabular}

Course evaluations showed that the students were less than "somewhat satisfied" with the organization of the course and the amount they learned in the course. The ratings were lower than normal for the instructor. One concern that emerged from written comments was that it is difficult to write a text about a topic area about which students have no previous knowledge. Another key problem was that it was difficult for the instructor to provide interim grades throughout the course, especially with respect to the editing. The instructor was reluctant to give too much feedback to writers because he wanted the students to exert their editorial roles. However, the editorial effort was often "hit or miss." Moreover, over time, it became more difficult for everyone to 
manage the writing process. MediaWiki does not support threaded discussion. Over time, students in the class found it increasingly difficult to post their comments and discuss the content of a wiki page in its discussion page. The students sensed a lack of control and accountability in such a collaborative setting. The first author realized that design opportunities emerged from these difficulties.

When the author of this paper took on the role of a Senior Editor in the class, she also realized that the collaborative writing process needs good governance in order to better organize the project. The implications that arose from this project are as follows.

First, because documents in a wiki are constantly evolving, we cannot conclusively determine when a document reaches its finished version; however, in mandatory collaborative writing, there is a need for a clear definition as to what the finished version will be. The implication was drawn from the class that we should have an official process in which group members agree on what the final version of a page should resemble.

Second, collaborative writing needs a process in which to standardize the way group members add topics and details in each article. Group members should use simple wiki markups to format text such as "*" for bullet, "\#” for number, and "==" for header. Fonts in some tables were too small or of widely varying sizes. A standard should be set for the table formatting and fonts for the sake of a harmonious appearance. Group members should reach a settlement on which formatting styles will be used throughout the writing process.

Third, as explained above, as the document evolves, the comment section grows more confusing. We attempted to solve this problem manually at that time by suggesting a format for comments. The header of each comment should provide the name of the person who provides the comment, the date the comment was posted, and key words to indicate whether the comment is classified as an Author's reply, an Editor-in-Chief Comment, or Senior Editor Feedback. The order of the comments should be the same, either from the most recent backward or vice versa.

Fourth, the Associate Editors should submit to the Senior Editor an executive summary that they edit each week in order to facilitate follow-up work. The Senior Editor's job then is to make a report to the Editor-in-Chief summarizing the work of the four pairs of Associate Editors. This report can also go on the main discussion page so the Associate Editors can review the reports easily the following week. This would make the reviewing process smoother.

Fifth, the Senior Editor had responsibilities from Sundays to Saturdays, but the class was held on Tuesdays. The Senior Editor each week could thus only report progress from Sunday to Tuesday. Therefore, at the beginning of each class, the Senior Editor from the last week should pass on the work to the current week's Senior Editor and brief everyone on the progress. The duties of the Senior Editors overlap two weeks, helping to maintain continuity in the review process.

Sixth, everyone should present the progress of their sections during the class and get comments from others. Communication will allow the Associate Editors to understand the authors' ideas and help them review their sections. The class should also budget some time in each class session for contemplating whether or not the current topics are appropriate and whether they fit in. Leaving them to the Senior Editor may be too overwhelming.

Seventh, because this was a mandatory writing process, the comments of the Associate Editors should be followed up to see if they were acted upon. If not, the Authors' reasons for not doing so should be given. This way Editors' efforts are not wasted.

Eighth, after the Secondary Authors have posted their work in the discussion page, who should take the responsibility of merging the content and when? That person should decide how it should fit in. Some alternatives were suggested, such as giving the First Author sole discretion in making 
such decisions, having the First Author consult with the Secondary Authors, or having the class decide upon this collectively.

Finally, there are some topics that were redundant in many sections. Some alternatives were suggested, for example, agreeing on who would take responsibility as a main author to gather content into one place in the book. Decisions may be made solely by the First Author or jointly with the Secondary Authors. If it is reasonable, there should be - at various places in the book - a link created so the reader can get comprehensive information. Redundancy should be avoided through discussion amongst authors about their own specific details.

\section{Problems Discovered}

Some problems that we faced in the project, we attempted to solve by utilizing good governance. However, several other difficulties emerged in using MediaWiki for this project:

1. Watchlists are a feature of MediaWiki to keep track of pages that are interesting to a user. Whenever these page change, the system will notify the user of the changes. However, this still requires the user to discover the specific changes that have been made. If they wanted to comment on changes to a page, they would use the Discussion page. Keeping track of changes to discussion pages added another layer of complexity.

2. The first author built a table (page) to overview the status of the entire project (see Figure 3 ). It became clear that it would be very useful if an entry in the "Discussion" page could automatically update the summary table. The table showed the status of a particular (group-authored) page as either "ready for writing", "ready for editing", "complete", or "not started". The "ready for writing" status would be set by an Editor who has completed an analysis of the page. The "ready for editing" status would be set by a writer who has completed edits to a page. It was decided that editorial notes should be placed last-in-first-out in the "Discussion" page. Notes were written alternatively by an Editor

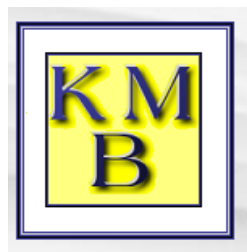
\begin{tabular}{l|l|l|l|l|l|l|l|} 
article discussion & edit history move
\end{tabular}

my talk preferences my watchlist my contributions log out KM Book Project Table Summary

\begin{tabular}{|c|c|c|c|c|c|}
\hline Sections and subsections & $\begin{array}{c}\text { 1st } \\
\text { author }\end{array}$ & $\begin{array}{l}\text { 2nd } \\
\text { authors }\end{array}$ & Status & $\begin{array}{c}\text { Last } \\
\text { updated }\end{array}$ & Comments \\
\hline 1. Introduction (A) & An & - & Complete & $11 / 20$ & - \\
\hline What is Knowledge? & An & $\begin{array}{l}\mathrm{Be}, \mathrm{M} \\
\mathrm{A}, \mathrm{E}\end{array}$ & In Progress & $11 / 22$ & - \\
\hline Tacit, Explicit \& Implicit & An & E & Complete & $11 / 22$ & - \\
\hline Expertise & - & - & Not Started & - & - \\
\hline Context & - & - & Not Started & - & - \\
\hline Knowledge Obsolescence & $\mathrm{Ke}$ & - & Complete & $11 / 26$ & - \\
\hline Intellectual Capital & Be & - & Complete & $11 / 15$ & - \\
\hline Protecting Knowledge & - & - & Not Started & - & - \\
\hline Legal Environment & - & - & Not Started & - & - \\
\hline Theories & - & - & Not Started & - & - \\
\hline Data, Information \& Wisdom & E & $\mathrm{M}, \mathrm{A}$ & Complete & $10 / 17$ & - \\
\hline Knowledge Cycle & Be & E & Complete & $11 / 22$ & - \\
\hline Philosophy of Knowledge & - & - & Not Started & - & - \\
\hline Quality of Knowledge & - & - & Not Started & - & - \\
\hline Evaluation of Knowledge & - & - & Not Started & - & - \\
\hline Knowledge Claim Evaluation & |Be & |- & Complete & $11 / 22$ & |- \\
\hline
\end{tabular}

Figure 3: KM book project table summary 
and a Writer. The type of note had a particular "heading" characteristic that would annotate the required changes or summarize recent changes.

3. While the summary table was useful, we also needed a tool to automatically populate and update it. For example, anyone who was interested in being the main editor of a specific topic in the KM book would start writing a page, and the mechanism would update the summary page by putting that person's name in the main author field, updating the status of that page whenever he or she makes some progress. Thus, the Senior Editor could start his or her work from this table before going into details of each topic.

4. Some issues with the writing process were organizational (e.g., maintaining motivation, developing formatting standards, determining protocols for work status, etc.) and could not be solved by adding technical processes to the wiki.

\section{Define Objectives of a Solution}

The focus of this paper is on the design of solutions to the weaknesses of MediaWiki when used as a group writing tool in a classroom setting. As a result of our experiences in the group writing tasks described above, the objective was to design processes to support some requirements of collaborative writing in higher education. Solutions to organizational problems require a redesign of the course process and are not addressed here. In this section we propose some solutions to the problems the class faced in performing tasks such as commenting and project management. We first discuss principles of group (collaborative) writing, outline other research and solutions related to these processes, and then specify requirements that can lead to improving these processes.

\section{Group (Collaborative) Writing in the Classroom}

According to Tammaro, Mosier, Goodwin, and Spitz (1997), "Writing is a complex process" (p. 21). Although the complexity and difficulty of writing tasks increase dramatically when more than one person is involved, there are many advantages to group collaboration, such as encouraging group members to work in cooperative ways to share knowledge and exchange ideas. As predicted by constructivist learning theory, group collaboration can overcome limitations of individual knowledge and skills (McCarthy, Miles, \& Monk, 1991). Individuals can create new content individually or with other group members and can help each other improve quality. Feedback from instructors is another factor that can encourage students to perform better (Barros \& FelisaVerdejo, 2000; H-J. Suh \& Lee, 2006). However, the disadvantages of group collaboration are the difficulty of coordinating a joint effort and the diversity of group members (McCarthy et al., 1991). In collaborative writing, it is difficult for members to follow the discussion and add their own points (Weng \& Gennari, 2004). Instructors also find it difficult to monitor, assess, and promote students' contributions and performance (H-J. Suh \& Lee, 2006).

Posner and Baecker (1992) developed a taxonomy that categorized roles, activities, control methods, and strategies in a collaborative writing project. They identified that each person in the group has at least one role: writer, consultant, editor, and reviewer. Collaborative writing is composed of several sub-processes: brainstorming, note taking, organizational planning, writing, revising, and editing. Posner and Baecker categorized control methods: centralized (only one person controls all documents in the project), relay (one person controls all documents at a specific time and control can pass among group members), independent (each person has responsibility for a separate part of the document and controls his or her own part), and shared (several group members have equal privileges to control the document at the same time). They also classified strategies of a collaborative writing project: single writer (one person writes the document but he or she may get the ideas from discussion with other members), scribe (only one person has the responsibility of writing the group's thoughts), separate writers (group members split the document into parts 
and each person takes responsibility for an individual part), and joint writing (group members collaborate and decide the content of the document and then write the document together).

The writing process is not static while the document is evolving, especially in a group setting. Beck and Bellotti (1993) conducted interviews, surveys, and case studies to analyze the coauthoring process. They concluded that the nature of a group is dynamic. Roles, goals, tasks, and strategies change over time as group members respond to external influences, which change circumstances and bring unpredicted events.

In higher education, collaboration is vital. Graduate students need to perform collaborative assignments, do research and case studies, as well as review, summarize, and critique papers. There are many ways to manage how students will perform collaborative writing. For example, Schulz and Ludlow (1996) developed group writing models in which the instructor allowed students to divide the topic into sections and let each student in the group individually take responsibility for finishing his or her own section. Another model is one in which the instructor singles out one student in the class to start writing on the assigned topic, while the other students in the group proofread and edit the content. It is vital for the instructors to design the model of group writing they wish to use and clearly inform students.

As can be seen from this review, the design of the pedagogy for a group writing class entails many choices by the instructor. It is not clear from the literature which choices to make. Moreover, the technical issues associated with facilitating the group writing process are not addressed by the literature.

\section{Related Research and Proposed Solutions}

Constructivism plays a vital part in defining how wikis can be used for collaborative writing. Ideally, the technology (e.g., a wiki) should not inhibit group writing tasks. Our experience indicated that it is necessary to improve discussion procedures since this is a crucial part in collaboration. From a technical standpoint, the students found it difficult to follow the discussion and add their own points because in MediaWiki, the content page and discussion page are separated. This also relates to workspace awareness (Liccardi, Davis, \& White, 2007, 2008a, 2008b). As the amount of content increases, members may find it is difficult to locate the section in the content that they want to discuss. Kriplean, Beschastnikh, McDonald, and Golder (2007) assert that MediaWiki needs a mechanism to provide an annotated version of conversations to keep better track of discussions: which participants were involved in the process, what had been discussed so far, and where to locate updates in the conversation on the discussion page. Designing a process to improve the discussion page would be beneficial for group members in a constructivist learning environment, so that they can easily comment, reply, argue, create threads of conversations, categorize discussions, keep track of content, and involve their peers in the decision and negotiation process. In this way, students can be at the center of the learning process and will be able to more easily monitor and direct their ability to construct knowledge.

One dominant characteristic of constructivism is learning as a social activity. Learning and cooperation involve an intimate connection to other group members. Knowing what tasks one's peers are engaging in while writing in the project provides awareness in social aspects by supporting conversation and interaction with other members. According to Kriplean et al. (2007), MediaWiki needs an awareness tool that can summarize tasks to better assist the agreement process among wiki members. Several studies have attempted to improve wiki workspace awareness and users' awareness to enhance the effectiveness of collaborative writing. One example is CAWS (CoAuthoring Wiki based System) (Liccardi, 2007; Liccardi et al., 2007, 2008a, 2008b), which aims to design co-authoring features in wiki-based systems to support the collaborative authoring system and increase awareness. CAWS focuses on four aspects of awareness: personal, social, in- 
formal, and group (Liccardi et al., 2008a). CAWS also provides an annotation system attached to threaded discussions, and users can filter comments by type or by author (Liccardi et al., 2008b). CAWS is in a stable stage, but it still lacks other functionality, such as instant messaging and a polling system (Liccardi et al., 2008b). While CAWS is based on the wiki concept, it is not implemented on the MediaWiki platform.

Collaborative writing may require a project management feature, which was one of the missing elements in MediaWiki. Student feedback in the course evaluations revealed declining motivation and action over time, a general sense of lack of control and accountability in the collaborative setting, and a lack of clarity in direction. All of these are useful points to justify why a project management feature would be useful. Additionally, from a constructivism perspective, this process provides awareness and motivation to students. They can see how much progress they have made when compared with their peers.

\section{Design Alternatives for MediaWiki Extensions}

We propose two processes that could be developed as extensions to MediaWiki: a discussion process for supporting an effective asynchronous discussion and a project management process for guiding students through the writing process.

\section{Discussion Process}

Weng and Gennari (2004) proposed an asynchronous collaborative writing system through annotations. We apply their model to design a discussion process for MediaWiki. The discussion process model (Figure 4) has two layers: interface and content. In the content layer, the evolving wiki document is composed of three contexts: page, text, and annotation. Students and instructors utilize the Discussion Tool in the interface layer to add threaded discussion or annotation in the wiki document. The activities from students and instructors are kept in the Discussion Database. The Awareness Tool calls the stored procedure in the Discussion Database to retrieve information from the Discussion Database and notify instructors and students based on different filters, such as annotations made by each user, annotation category, and newly-added annotations in the specified period of time. The Discussion Database collects context (metadata of the content that an annotation is attached to, i.e., the page, text, and annotation context), message body (the actual text of the annotation), annotation creator, annotation recipient, annotation time and date, response (used in threaded discussions), status (what happened to a specific annotation), voting (most dissatisfied to most satisfied), and urgency (the priority of annotations) (Weng \& Gennari, 2004).

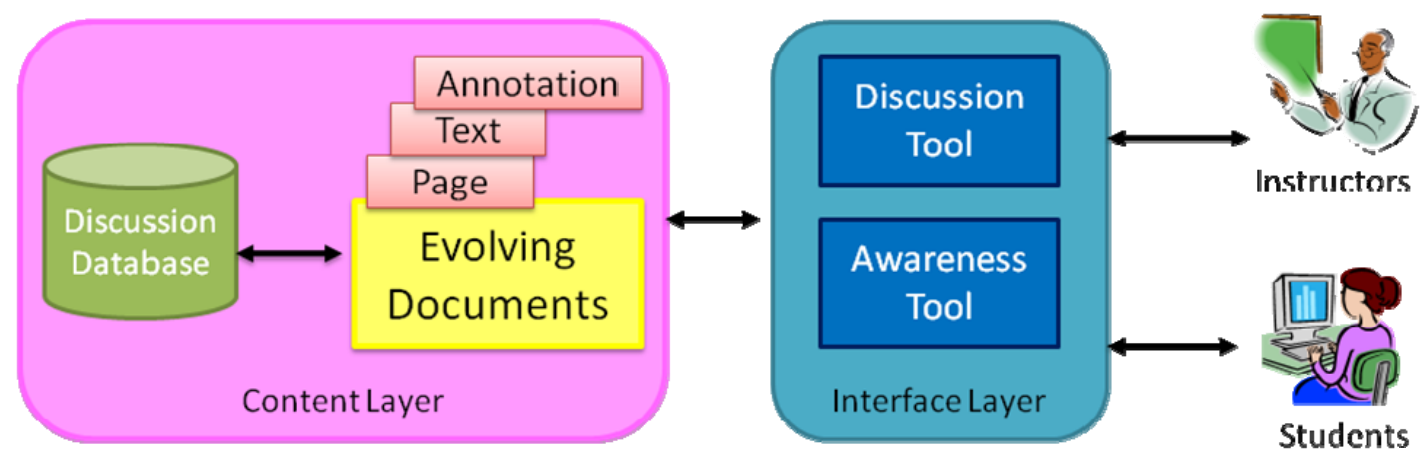

Figure 4: Discussion process (Adapted from Weng and Gennari, 2004) 
As noted above, MediaWiki does not have a good discussion system. Users have to go to a discussion page to add comments. These comments can be placed at any section of the page and are not automatically sorted in chronological order. The more students involved in a discussion, the more comments are posted, and it quickly becomes difficult to track and organize comments. Also, without an awareness system, it is difficult for users to identify what has been changed, who has made the changes, and when those changes were made. Our proposed design will provide a discussion area on the same page with the wiki content page. The process could work as follows:

A student starts the first discussion thread by choosing a paragraph, selecting what category this thread should be sorted under (i.e., suggestion, agreement, disagreement), and designating the importance of the thread. Other students can reply to this discussion and rate each comment (by approval or importance). Those who contribute to the discussion thread have the ability to edit or delete their content. Whenever anyone creates or replies to a discussion thread, the system will update the data in the database and notify all participants.

\section{Project Management Process}

According to Bean and Hott (2005), there are some wiki providers, such as Socialtext (http://www.socialtext.com) and JotSpot (http://jot.com) that have project management features. These leading enterprise wiki vendors provide an extensive, complex system for enterprise wikis, which have license fees. B. Suh et al. (2008) proposed two types of WikiDashboard - Article Dashboard and User Dashboard - for live Wikipedia pages. The WikiDashboard provides a graph that presents information about how many revisions have been made on each Wikipedia page and who edits this Wikipedia page. This WikiDashboard displays on the top of each Wikipedia page, which allows users to easily assess the status of a page.

The project management process (Figure 5) retrieves an interaction user log from the Performance and Discussion Databases to create a Project Status Summary Database. The process queries the Project Status Summary Database to display the status of each page on the top of the wiki page and automatically updates the table summary of the project. In the summary page a table displays an overall picture of the project, such as who the first author is, who the second authors are, when last modified and by whom, as well as current status of this page (i.e., not started, being edited, updated on, not yet updated, requires more review, and completed).

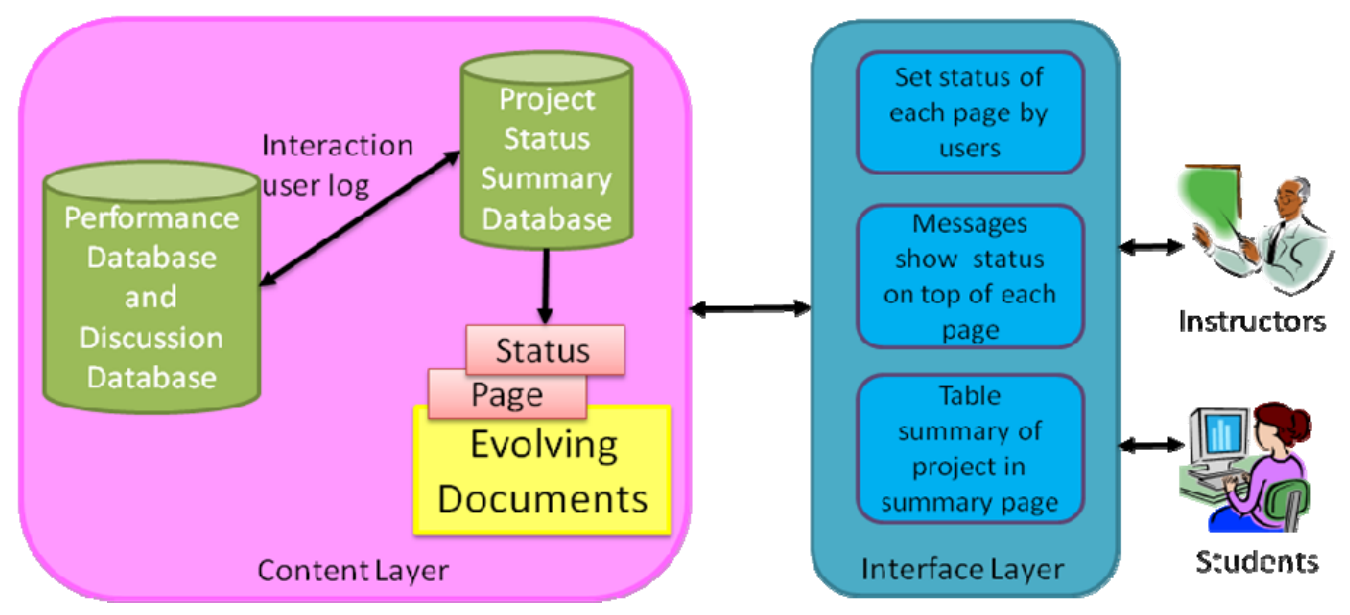

Figure 5: Project management process 
Authors and editors can modify the status of each page (e.g., ready for writing or ready for editing) and it is recorded in the Project Status Summary Database. They can make changes to finalize the status of the page, but we limit changing status only for authors. The Editor can suggest or discuss the page in the Discussion Tool based on the status, which is shown on the top of the page.

\section{Preliminary Demonstration and Evaluation}

According to Hevner et al. (2004), design science research is composed of an iterative build-andevaluate loop. We did a preliminary demonstration and evaluation of our designs by discussing the project management process with the KM class. The model of the project management process is shown in Figure 3. After we built the summary table, students in the class provided positive feedback and agreed that this was a useful tool in tracking the overview of the KM book.

For the discussion process, we conducted a preliminary demonstration and evaluation with the focus group in a different doctoral seminar class, using semi-structured interviews. There were nine graduate students in the focus group. Three of them were female and the other six were male. All of the students in the focus group were familiar with using wiki technology. The whole process of conducting focus groups lasted approximately one and a half hours.

We used a set of interview questions adapted from Patton (1990) in the focus group session. The first question was, "Which of the collaborative tasks (construction of learning object and production of written paper reviews) were beneficial to your learning process? And why?" A summary of their feedback is as follows.

First, construction of learning objects is more beneficial to learning when working in a group. In that context, a learning object was a group activity that focused on analyzing reading materials in a group and posting it on a MediaWiki. One student liked learning objects, but wished for a private space to write. She also felt like she could not write as much as she would have liked because she did not know how much or how little her classmates had contributed. Some students felt the learning process was better but more time consuming with the learning objects in place.

Second, reviewing the writings of others helps the learning process. Working with others allows a degree of flexibility, in that one can focus on subjects he or she is confident in, while others can aid in subjects that they themselves are confident in. A student suggested dividing each group into two subgroups: one subgroup dedicated to writing and another to reviewing. Some students felt that there should be alternating cycles of writing one week and then reviewing the next. Students suggested that with a large enough group, students could have a cycle of studying and gathering material one week, taking that material and writing the next week, and reviewing the next. Two students liked to rotate team members every week to have time to work with other people. However, while they said they were amenable to learning at the beginning of the semester, they also believed that they had started to lag towards the end of the semester.

Finally, since the roles in groups were in rotation, one student said he enjoyed the opportunity to occasionally become the team leader. Whoever is the team leader will have to know the personalities of their team members and how to best manage them. The difficult part of being a team leader is getting his or her fellow students to work, communicate, and provide feedback on a timely basis. A student suggested that the class should let one person in the group with a particular interest in a certain subject become team leader for that section of the review process.

The next question we asked interviewees was, "Did you experience any technical difficulties while communicating with your team members? If so, which ones?" We learned that students communicated with their group members through face-to-face discussion in and outside class and through Email. One student said Email works as a communication tool, but not as a collaborative 
tool, as nobody else besides the sender and recipients can see the communications. Some students communicated through notes in the wiki on the discussion page and through these notes other team members could see the back-and-forth communications between them.

We demonstrated the model of our discussion process designs to the participants in the focus group and explained how they function. Then we provided examples of a variety of user interface prototypes to them: collaborative protocol reviewing (Weng, Gennari, \& McDonald, 2004), which provided threaded discussions with an awareness system; CommentPress (The Institute for the Future of the Book, n.d.), which provided an innovative interface with a threaded discussion; and a mouse over interface, which displayed a dialog box from which users could see and add comments directly in the box. We explained how each of these prototypes function. We also showed how the current discussion page of MediaWiki works because some members of the focus group were not using discussion pages.

We then asked the following questions: "What do you like and/or dislike in this new design? How do discussions in this new design differ from discussions in general? And what improvements to the discussion page in MediaWiki do you think can help you collaborate and discuss with your partners better? Why?" A summary of their feedback follows.

Students liked the various designs because they provided an easier way to keep track of what they and their peers had done. The students suggested a way to track changes made by different authors, like the Track Changes feature in Microsoft Word. Some students suggested a private space to keep drafts before posting them in the public space. Further, some students wanted the ability to comment privately. One student liked the mouse-over interface, but also liked the idea of displaying the topic of each section as in CommentPress.

Based on this evaluation, we found that our proposed design would be useful to students in graduate courses. Most participants preferred a simple user interface that provided enough information for them to use in a discussion.

All participants in our focus groups have given us positive feedback and suggested ideas to improve the design of the interface. Based on the feedback that we received, our next step is to develop the prototype based on our proposed design, conduct a usability test and test the efficiency of the first prototype, assess whether it is appropriate in a classroom setting, and gather feedback to redesign and improve the processes.

\section{Conclusion}

Our study contributes to design science. It has followed the DSRM process model for information systems research (Peffers et al., 2007). The problem situation surfaced as a result of using a wiki to support collaborative writing on a mandatory basis. In this paper, we propose two processes designed to support collaborative writing in higher education systems using MediaWiki: 1) a discussion tool based on Weng and Gennari (2004), which changes the way people discuss content and increases group awareness; and 2) a project management tool, which increases awareness of the status of each part of the writing project. These designs that we proposed in this study are only a subset of those that we think are important. There is still much room for improving the functionality and features of wikis to foster the collaborative aspects of learning.

One limitation of this research is in fulfilling just some of the requirements of DSRM. It is not a complete design, as it is only iteration and it lacks a complete evaluation. According to Hevner et al. (2004), the nature of good design is iterative. The process (set of activities) of creating the product (an artifact) that can address a business need and solve a problem is usually to go back to the design stage again and again after the designed artifact is tested, used, and observed. We plan 
to build the proposed plug-in prototypes and evaluate their effectiveness and usability. Further study will be needed to redesign better processes.

Knowledge is a valuable asset in both business and academia. The real value in knowledge management lies in sharing ideas and insights as tacit knowledge. Interaction and collaboration play an important role in the transmission of tacit knowledge within a group, or the conversion of tacit knowledge into explicit knowledge, leading to knowledge sharing at the organizational level (Nonaka, 1990, 1994; Nonaka \& Takeuchi, 1995). According to Brockway (1996), many organizations spend time and money ensuring the appropriate use of knowledge technologies. If wellmanaged, these technologies will become valuable assets to the enterprise. Technology-based knowledge construction is meaningless if it cannot facilitate the translation of social elements of corporate life and culture into a digital format. As such, the technology involved in this context must be intuitive and easy to accept and use for end-users. At minimum, it must provide a quick and simple way to input information because the easier it is for content owners and site contributors to manage their content, the more likely they will be to do so. MediaWiki is a useful tool for supporting group collaboration. However, when we apply MediaWiki to the academic setting, we need to consider and adapt some features to match the needs of the classroom environment, which requires mandatory collaborative writing. The major success factor of using wikis in collaborative work is the motivation of members. Wiki technology can promote the collaboration environment and enhance communication and knowledge sharing; however, the vital part originates from group members themselves who have the responsibility to achieve the goals of mandatory collaboration in group writing. With assistance from appropriate planning and organizing, using wikis for collaborative writing can produce effective learning outcomes with the addition of some new tools.

Moreover, our research contributes to transdisciplinary research. Cohen (2009) calls transdisciplinary research Informing Science and defines it in terms of a meaningful set of research aspects that are shared by various disciplines (management information systems, library and information science, communication theory, education science, etc.). The Informing Science framework is composed of three components: the Informer, the Delivery System (information processing and transmission, what is otherwise called information system), and the Client (receiver of information). In our study, the Informer is the student (the author of the KM book). The Client refers to the student classmates (Associate Editor and Senior Editor) and the instructor (Editor-in-Chief). The Delivery System is the MediaWiki platform that supports collaborative writing in the classroom. The context or environment of the Informer and Client is the organization of the classroom, with its specific processes, goals, limited timeline, and dynamic roles and responsibilities. Another part of the context/environment is the needs of the Informer and the Client related to accomplishing their collaborative work. Our study contributes to informing science by demonstrating how the organization and needs of the Client and the Informer influence the design evolution of the system investigated.

Furthermore, our study suggests that there are mutual influences between the organization of classroom and the MediaWiki system. Exposed to specific aspects and constraints of the system, the students were found to be less than somewhat satisfied with the organization of the course. This can be interpreted from the perspective of the Information View of Organizations (IVO) (Travica, 2005). IVO is a new conceptual framework that focuses on studying organizations from an IS viewpoint and focuses on interactions and mutual influences between IS and organization. IVO suggests that the students need to be indoctrinated into a new information culture or infoculture (Travica, 2005, 2008) in the course of the gradual system development. Specifically, a new infoculture would need to balance the value of ensuring autonomy for editorial work of students with practices of providing effective interim feedback on students' work. 
Our study also confirms Henderson and Venkatraman's (1993) strategic alignment model between the business domain and the IT domain. Their model demonstrated two needs. The first of these is for alignment between business strategy and IT strategy. The second is for alignment between organization infrastructure and processes, and information systems infrastructure and processes. To successfully convert strategy into infrastructure and processes, we need both organizational design and information systems design activities (Henderson \& Venkatraman, 1993).

While we do not claim that we have exhausted all possible process designs to support collaborative writing, we believe that our proposed design provides an interesting perspective on how discovering problems in pedagogies leads to the design of innovative IT artifacts, i.e., digital learning objects. We hope that other researchers will find our designs useful in developing extensions for MediaWiki to fulfill the goals of mandatory collaborative writing.

\section{References}

Alavi, M. (1994). Computer-mediated collaborative learning: An empirical evaluation. MIS Quarterly, $18(2), 150-174$.

Barrett, D. J. (2009). MediaWiki. Beijing: O'Reilly.

Barros, B., \& FelisaVerdejo, M. (2000). Analysing student interaction processes in order to improve collaboration: The DEGREE approach. International Journal of Artificial Intelligence in Education, 11, 221-241.

Bean, L., \& Hott, D. D. (2005, July/August). Wiki: A speedy new tool to manage projects. Journal of Corporate Accounting \& Finance, 16(5), 3-8.

Beck, E., \& Bellotti, V. (1993). Informed opportunisms as strategy: Supporting coordination in distributed collaborative writing. In C. S. G. De Michelis, \& K. Schmidt (Eds.), Proceedings of the European Conference of Computer-Supported Cooperative Work (pp. 233-248). Dordrecht: Kluwer.

Bouton, C., \& Garth, R. Y. (1983). Learning in groups. New Directions for Teaching and Learning Series (Vol. 14). San Francisco: Jossey-Bass.

Brockway, D. W. (1996). Knowledge technologies and business alignment. Information Management \& Computer Security, 4(1), 45-46.

Bruner, J. S. (1996). The culture of education. Cambridge: Harvard University Press.

Cohen, E. B. (2009). A philosophy of informing science. Informing Science: the International Journal of an Emerging Transdiscipline, 12, 1-15. Retrieved from http://inform.nu/Articles/Vol12/ISJv12p001015Cohen399.pdf

Cunningham, W. (2006). Design principles of Wiki: How can so little do so much? Retrieved February 20, 2008, from http://c2.com/doc/wikisym/WikiSym2006.pdf

De Pedro, X., Rieradevall, M., López, P., Sant, D., Piñol, J., Núñez, L., et al. (2006). Writing documents collaboratively in higher education using traditional vs. wiki methodology (I): Qualitative results from a 2-year project study. Proceedings of the 4th International Congress of University Teaching and Innovation, Barcelona, Spain. Retrieved February 14, 2008 from http://uniwiki.ourproject.org/Article+Wikis

Dewey, J. (1910). How we think. Boston: D. C. Heath.

Dewey, J. (1916). Democracy and education. New York: The Free Press.

Harasim, L. (1990). On-Line education: Perspectives on a new medium. New York: Praeger/Greenwood.

Henderson, J., \& Venkatraman, N. (1993). Strategic alignment: Leveraging information technology for transforming organizations. IBM Systems Journal, 32(1). 
Hevner, A. R., March, S. T., Park, J., \& Ram, S. (2004). Design research in information systems research. MIS Quarterly, 28(1), 75-105.

Hiltz, S. R., Coppola, N., Rotter, N., \& Turoff, M. (1999). Measuring the importance of collaborative learning for the effectiveness of ALN: A multi-measure, multi-method approach. In J. Bourne (Ed.), Proceedings of the 1999 Sloan Summer Workshop on Asynchronous Learning Networks: Online education Vol. 1. Learning effectiveness and faculty satisfaction. (pp. 101-120). Sloan-C.

The Institute for the Future of the Book. (n.d.). Gamer Theory. Retrieved February 14, 2009, from http://www.futureofthebook.org/gamertheory/?cat=5

Kriplean, T., Beschastnikh, I., McDonald, D. W., \& Golder, S. A. (2007). Community, consensus, coercion, control: CS*W or how policy mediates mass participation. Proceedings of the 2007 International ACM Conference on Supporting Group Work, 167-176.

Liccardi, I. (2007). CAWS: Improving users' awareness in collaborative authoring activities. Proceedings of the Conference on Supporting Group Work Group '07 Doctoral Consortium papers, Sanibel Island, Florida.

Liccardi, I., Davis, H. C., \& White, S. (2007). CAWS: A wiki system to improve workspace awareness to advance effectiveness of co-authoring activities. Proceedings of the Conference on Human Factors in Computing Systems CHI '07, San Jose, California, USA, 2555 - 2560.

Liccardi, I., Davis, H. C., \& White, S. (2008a). CAWS: An awareness based wiki system to improve team collaboration. Proceedings of the eighth IEEE International Conference on Advanced Learning Technologies, Santander, Cantabria, Spain, 265-267.

Liccardi, I., Davis, H. C., \& White, S. (2008b). CAWS: Visualizing awareness to improve the effectiveness of co-authoring activities. Special Issue of Collaborative Computing in IEEE Distributed Systems Online.

McCarthy, J., Miles, V., \& Monk, A. (1991). An experimental study of common ground in text-based communication. Proceedings of the SIGCHI Conference on Human Factors in Computing Systems: Reaching through Technology, New Orleans, Louisiana, USA, 209-215.

Nonako, I. (1990). Redundant, overlapping organization: A Japanese approach to managing the innovation process. California Management Review, 32(3), 27-38.

Nonako, I. (1994). A dynamic theory of organizational knowledge creation. Organization Science, 5(1), 1437.

Nonaka, I., \& Takeuchi, H. (1995). The knowledge-creating company: How Japanese companies create the dynamics of innovation. New York: Oxford University Press.

Parker, K. R., \& Chao, J. T. (2007). Wiki as a teaching tool. Interdisciplinary Journal of Knowledge and Learning Objects, 3, 57-72. Retrieved from http://ijello.org/Volume3/IJKLOv3p057-072Parker284.pdf

Patton, M.Q. (1990). Qualitative evaluative research methods. Thousand Oaks, CA: Sage.

Peffers, K., Tuunanen, T., Rothenberger, M. A., \& Chatterjee, S. (2007). A design science research methodology for information systems research. Journal of Management Information Systems, 24(3), 45-77.

Posner, I. R., \& Baecker, R. M. (1992). How people write together. Proceedings of the Twenty-Fifth Annual Hawaii International Conference on System Sciences, Kauai, Hawaii, USA, 4, 127-138.

Raman, M., Ryan, T., \& Olfman, L. (2005). Designing knowledge management systems for teaching and learning with wiki technology. Journal of Information Systems Education, 16(3), 311-320.

Richardson, W. (2006). Blogs, Wikis, podcasts, and other powerful Web tools for classrooms. Thousand Oaks, CA: Corwin Press.

Schulz, K. H., \& Ludlow, D. K. (1996, July). Incorporating group writing instruction in engineering courses. Journal of Engineering Education, 85(3), 227-232. 
Suh, B., Chi, E. H., Kittur, A., \& Pendleton, B. A. (2008). Lifting the veil: Improving accountability and social transparency in Wikipedia with WikiDashboard. Proceeding of the Twenty-Sixth Annual SIGCHI Conference on Human Factors in Computing Systems, Florence, Italy, 1037-1040.

Suh, H.-J., \& Lee, S.-W. (2006). Collaborative learning agent for promoting group interaction. Electronics Telecommunications Research Institute (ETRI) Journal, 28(4), 461-474.

Tammaro, S. G., Mosier, J. N., Goodwin, N. C., \& Spitz, G. (1997). Collaborative writing is hard to support: A field study of collaborative writing. Computer Supported Cooperative Work: The Journal of Collaborative Computing, 6, 19-51.

Travica, B. (2005). Information view of organization. Journal of International Technology and Information Management, 14(3), 1-20.

Travica, B. (2008). Influence of information culture on adoption of a self-service system. Journal of Information, Information Technology, and Organizations. 3, 1-15. Retrieved from http://jiito.org/articles/JIITOv3p001-015Travica470.pdf

University of Delaware. (2008, May 23). Wiki in higher education. Newark, DE: University of Delaware, IT-User Services. Retrieved October 15, 2008, from http://udel.edu/ mathieu/wiki/resources/2008-523 Wikis_in_Higher_Education_UD.pdf

Vygotsky, L. S. (1978). Mind in society. Cambridge: Harvard University Press.

Wegerif, R. (1998). The social dimension of asynchronous learning networks. Journal of Asynchronous Learning Networks, 2(1), 34-49.

Weng, C., \& Gennari, J. H. (2004). Asynchronous collaborative writing through annotations. Proceedings of the 2004 ACM Conference on Computer-Supported Cooperative Work, Chicago, Illinois, USA. 578581.

Weng, C., Gennari, J. H., \& McDonald, D. W. (2004). A collaborative clinical trial protocol writing system. In M. Fieschi et al. (Eds.), Proceedings of the $11^{\text {th }}$ World Congress on Medical Informatics (pp. 1481-1486). Amsterdam: IOS Press.

Wiki Design Principles. (n.d.). Retrieved February 15, 2008, from http://c2.com/cgi/wiki?WikiDesignPrinciples

\section{Biographies}

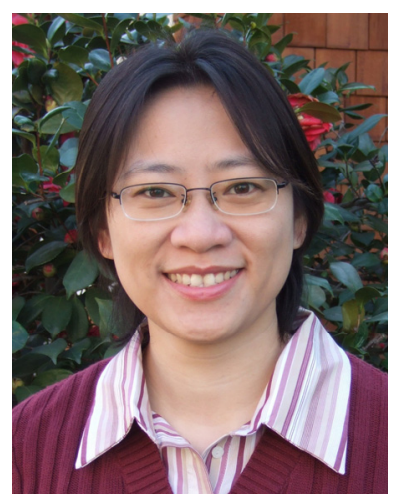

Sumonta Kasemvilas is a PhD student and a research assistant in the Social Learning Software Lab ([SL]2]) at the School of Information Systems and Technology (SISAT), Claremont Graduate University (CGU). Her research interests are Web and Internet technologies, online intellectual communities, group collaboration, and knowledge management. Her dissertation research addresses the design and development of extensions for wikis to support group collaboration and knowledge sharing in the classroom setting. 


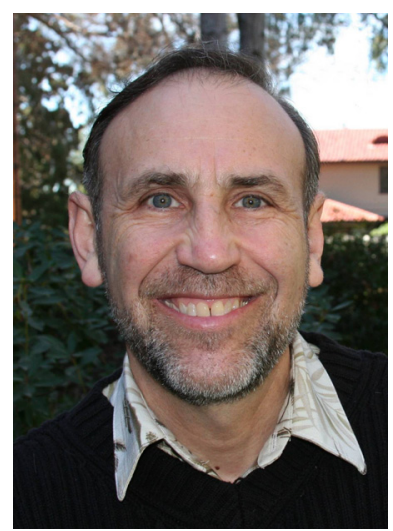

Lorne Olfman is a Professor in the School of Information Systems and Technology and Fletcher Jones Chair in Technology Management at Claremont Graduate University (CGU). He came to Claremont in 1987 after graduating with a PhD in Business (Management Information Systems) from Indiana University. Lorne's research interests include: how software can be learned and used in organizations, the impact of computer-based systems on knowledge management, and the design and adoption of systems used for group work. Along with Terry Ryan, Lorne co-directs the Social Learning Software Lab ([SL]2). A key component of Lorne's teaching is his involvement with doctoral students; he has supervised 45 students to completion. Lorne is an active member of the Information Systems community. 